\title{
LIFEREC: A Framework for Recommending Users from Past Life Experiences
}

\author{
MOHSIN ALI MEMON*, SANIA BHATTI*, SHAHZAD NIZAMINI*, NAEEM AHMED MAHOTO* \\ AND FARIDA MEMON**
}

RECEIVED ON 05.09.2016 ACCEPTED ON 21.02.2017

\begin{abstract}
Life logging has been an eminent topic of concern in recent years with many researchers focusing on capturing daily life activities of human. With the proliferation of IoT (Internet of Things) domain, the devices are now able to record human interaction for longer periods as well as transfer this data easily to other computing devices or cloud storage. This article proposes a novel framework named as LIFEREC which acquires information from Io $T$ aware devices and sensors. It maintains activity profiles of various activities performed by the users in their daily lives. Furthermore, the framework provides recommendations when requested by an individual while taking into account the past life history and current context. Recent research on digitizing human life is quite efficient in amassing enormous data but futile in offering assistance for prospect decisions in life. The data gathered by the lifelog devices may be of a great help in taking decisions. The proposed system gives a new direction to existing mechanisms of providing recommendations by exploiting the current context and the past experiences of human life. The recommendations provided by our proposed system may be very helpful while performing those activities which have already been experienced in the past.
\end{abstract}

Key Words: Lifelogging, Internet of Things, Recommendation.

\section{INTRODUCTION}

$\mathrm{L}$

ifelogging has become popular with the invention of cost effective digital devices for capturing information as well as amassing huge storage.

The term 'Lifelog' refers to the unprompted recording of everyday events of human life such as the activities performed by a person that may later be recalled or reminisced [1]. Some of those activities involve interaction with the IoT aware devices like smart TVs, vehicles, etc. while rest of the activities are physical in nature such as meeting with someone, sleeping, eating, site seeing, etc. Today, many projects like Memex vision [2] and Insense [3] exist that capture information for the life time and almost all of them aggregate massive amount of lifelogs which are intricate to filter, categorize and retrieve at particular moment. To make the best use of past lifelogs, the user must be provided with a system which may provide recommendations at a particular moment based on the current state. This research proposed LIFEREC, a novel lifelog framework which addresses the above mentioned challenges while

* Department of Software Engineering, Mehran University of Engineering \& Technology, Jamshoro.

** Department of Electronics Engineering, Mehran University of Engineering \& Technology. Jamshoro.

Mehran University Research Journal of Engineering \& Technology, Volume 36, No. 3, July, 2017 [p-ISSN: 0254-7821, e-ISSN: 2413-7219] 
remaining extendible and well-organized. It is important to note that the focus of this research is on the collection of activity lifelogs and retrieval of recommendations based of those lifelogs, and therefore, we do not focus on privacy concerns raised during these processes.

In the IoT domain, the devices are connected with one another as well as able to send the data collected by them over a period of time to another location. The interactions with such devices can be recorded along with the identification of the person who is responsible for these interactions. These interactions are actually the lifelogs, which may help to recommend the user in their future life. In olden days, people used to keep records of their lives in the form of hand written notes, diaries and photographs. But, with the invention of IoT aware devices, the user's burden of manually keeping the records has been shifted to these devices.For example, the television can sense your presence and keep the record of the types of programs you view on it. In another example, your personal vehicle keeps the track of all your mobility and sends your frequent paths to the server for record keeping. Apart from recording, these devices are able to communicate over the networks, thus making them a reliable source of data collection and distribution.

Apart from, IoT aware devices that are able to detect the activities of a user, there are several other activities that are sensed using wearable devices and body worn sensors. Activities such as eating, sleeping, meeting with someone may require sensors like accelerometer, RFID (Radio Frequency Identification) and Bluetooth, etc. Furthermore, the data generated by such sensors has to be moved to a central location by connecting them with laptops, smartphones, etc.

The exploitation of precious past events for existing situation of the user has been given less attention in the recent research. One of the major reasons behind the lack of employing past life data for making decisions for the current state is the lack of contextual information about the past life events. The data collected in the existing form is unusable for generating recommendations. Moreover, the lifelogs are stored in multiple devices and need to be relocated at one place in order to produce recommendations for the present state of the user. Most of the current lifelogging devices [4-6] do not consider the users' current context while capturing and have limited storage capacity.

The proposed framework allows confinement of daily activities of human life by IoT aware device as well as by body worn sensors, thus not restricting the lifelogs to be captured by a single device. The lifelogs are then gathered at the central server where they are utilized for recommending the users according to their current state.

We have organized the paper as following. The related work is discussed in section 2. Section 3 highlights the design considerations and Section 4 provides the proposed LIFEREC framework. Section 5 explains the methodology. Finally, Section 6 concludes the work.

\section{RELATED WORK}

Several researchers have attempted to digitize human life activities either using camera or body worn sensors and ended up with piles of images, videos and activities of users. The works of [2] and [7] are a good illustrations of capturing lifelogs via sensors such as, camera, accelerometer and GPS. Chen et. al. [8] developed a system named as iCLIPS to search images and videos captured with Microsoft sensecam [9] by taking several inputs from user such as location, date/time, weather and people around at the time of capture. Rawassizadeh et. al. [10] proposed a sharing model for lifelog data and recommended security policies to be implemented by any lifelog tool in order to protect significant life bits. Lee et. al. [11] used smart phones for collecting life log in the 
form of (Global Positioning System), photos, songs, SMS (Short Message Service) and weather information and utilized keyGraph [12] for identifying meaningful context from captured data. The efforts by these researchers lack the mechanism to recommend the users by utilizing their past lifelogs. Furthermore, the interface provided to interact with the past life requires the user to fill in various context to fetch the lifelogs.

Talking about the activities captured using lifelog device, an application named as Activity Designer was developed by [13] in which users were able to develop their own activity models manually and monitor daily activities such as fitness exercises, transport usage and other social activities. The system was a low cost ubiquitous computing application for gazing into past activities. Another application called the Digital Parrot [14] was designed to run on small mobile devices and maintains context aware semantic information to support people in remembering past events. Satchell et. al. [15] discussed the use of cell phone camera for documenting of every day events and sharing of real time images via MMS (Module Management System) and highlighted the impact of creating personal news and publishing for others to have insight of one's life. Since the context information was lacking while detaining the activity logs, therefore, it was almost impossible to use the lifelogs for recommendation purpose.

The authors [16-17] attempted to make a lifelog system that employs the current context of the user such as people and objects nearby along with the location. The system fetched past lifelogs based on the current context but did not provide any recommendations. The authors evaluated the system by comparing it with Vicon Revue lifelog camera. Our proposed framework takes several previous designs in to account and attempts to create a system that is extensible and helps the users in their day to day activities.

\section{DESIGN CONSIDERATIONS}

Lifelogging has several merits besides self-awareness and health consciousness. One of them is that the users may be provided with the recommendations in their prospect life based on their past activities. So far, the lifelogging devices available in the market are unable to capture each and every activity of the user. Moreover, the data collected by these devices is not organized in a fashion which could help the users to take instant decisions based on their past life experiences.

The purpose of proposing the lifelog recommendation framework is to provide a uniform approach of collecting lifelogs and employing them while performing similar activities in future. It is therefore, important to take some design considerations into account while developing the framework which can perform dual functions of recording daily life activities and providing recommendations. Following are the design considerations which must be considered while developing the lifelog recommender system.

\subsection{Authenticity of Lifelogs}

The first and most important element which should be given the priority while making the lifelog systems is the authenticity of lifelogs. Since the lifelogs are being captured autonomously, all the actions of the users are being captured in a continuous fashion. In our proposed system, the lifelogs captured by the IoT aware devices and sensors are transferred to the authorized smart device of the user. This step authenticates the personal lifelogs of the user. The authenticated data collected from various devices and sensors is then uploaded to a cloud server. Once the lifelogs are stored on the cloud server, they can be organized and processed in order to be used for future recommendations.

Mehran University Research Journal of Engineering \& Technology, Volume 36, No. 3, July, 2017 [p-ISSN: 0254-7821, e-ISSN: 2413-7219] 


\subsection{User Intervention}

The lifelogs are generated by the actions of the users while the sensors are always running in the background recording those actions. Thus, the IoT based devices and sensors keep the track of the user continuously without user's administration. However, the users must have appropriate control over the captured lifelogs so that if they fearabout any privacy infringement, they are able to obliterate that part of lifelogs. In the proposed system, the authorized smart device is used to provide an interface that may intervene the lifelogs and allow users to make alterations to the data being sent to the cloud server.

\subsection{Storage Requirement}

Storage is significant when it comes to lifelogs as they keep piling up while the user is performing the activities. It is important to keep these lifelogs at a place where they can be easily retrieved. Storing the lifelogs on the personal devices of the user will not be a decent option as they have limited and volatile memory. It will be therefore, more efficient to store this large collection of lifelogs at a cloud storage for easy and quick retrieval. In the proposed framework, the IoT based devices as well as sensors attached to the user body send the lifelog data to the personal device of the user, from where it is eventually forwarded to the cloud server.

\subsection{Versatility of Recommendations}

The data collected while capturing the activities of the user over a period of time can be used in many different domains. The lifelogs may be only utilized properly by providing recommendations to the user from their past. This aspect of exploiting the lifelogs beyond treating dementia patients [17-20] has been recently brought forward. Researchers have attempted to utilize lifelogs captured by the devices to create recommendations for future activities $[17,21]$. While recommending the users, the present context of the user may assist in recognizing the analogous past information. The proposed framework detects various activities of the user along with the current context and relates the lifelogs with this contextual information. Thus, when the user performs the same activities again, the previous experiences are retrieved from the lifelogs of the user and recommendations of future actions are generated to provide assistance to the user.

\section{LIFEREC FRAMEWORK}

Keeping the design considerations in view, we have developed the LIFEREC framework as shown in Fig. 1. The proposed framework will not only help to keep the precious life moments of the users organized, but will also assist the users in making decisions in similar situations encountered in future. Auser communicates with the lifelog cloud server via an authorized smart device. This device can be anything from tablet pc, laptop, smart phone, etc. for sending the captured lifelogs to the server and receiving back the recommendations based on present context of the user. The role of the lifelog server is to analyze individual activities and lifelogs, organize them in chronological and spatial order and store them in the form of activity profiles. The activity recommender complies requests from registered user devices only, so as to assure secure reclamation of life log information with the clients.

One of the design requirements for this framework was to create a flexible architecture, therefore, the proposed framework provides the users with the option to gather their lifelogs from any IoT based device or sensor and sent them to the cloud server. Since, the activity detection phase captures a variety of information and provides sufficient context of the user, the same context can be used to recall past experiences and get recommendations from the cloud server. As it has been stated before that, our research focus is on the collection of lifelogs and processing recommendations from past life data, therefore, the privacy of user lifelogs is out of the scope of this framework. 


\subsection{IoT Based Devices}

The act of making the devices able to communicate over internet and develop rich applications is termed as IoT [22,23]. The key aspects of IoT devices that need to be tackled carefully are heteroginity, connectivity, device naming, device addressing and security [24]. The functionality of these devices can be fully employed to generate and store lifelogs of individual users which may be sent to a central server. We will discuss a few of the IoT devices which are commercially available in today's market.

\subsubsection{Smart Fridge}

Smart fridge is the keyword used for a fridge that is capable of ordering groceries by itself according to the user's needs. The fridge keeps tracks of the items consumed by the user at a particular time and keeps them ready for consumption like chilling the cold drink can or defrosting the frozen items. The fridge also retains the knowledge about food allergens contained in the products and as well as their expiry dates. The use of RFID ((RadioFrequency Identification) antennas makes the fridge capable to perform the above mentioned tasks very easily [25]. The smart fridge can send all the interactions performed by the user to their authorized lifelog device. This data can be used for providing healthy recommendations to the user on their next interaction with the fridge.

\subsubsection{Smart Television}

Smart televisions are gaining popularity since they behave like a computer with internet connected, thus allowing them to view digital content directly and store the history of the content watched by the user. Furthermore, camera, microphone and NFC (Near-Field Communication) tags are used for identification of the user accessing the smart television [26]. In such case, the user may leave digital footprints that may be employed intelligently to provide recommendations based on the past viewed programs. The lifelog application may incorporate the current context such as free time available, user's mood and people accompanied by the user to provide appropriate recommendations.

\subsubsection{Smart Car}

Smart cars have been a question of debate since the last decade. Smart technologies like ITS (Intelligent Transportation Systems) and ADAS (Advanced Driver Assistance Systems) [27] have paved the way for making semi-autonomous cars. These cars have become context aware by perceiving various details around the car, such as, traffic monitoring, car engine, speed as well as frequently visited locations [28]. The data gathered by the smart car may infer about the driver's interest such as the driving skills, preferred paths and most valued places in the vicinity. The lifelog server storing this information may provide valuable recommendations depending upon the user current situation. For example, the user is suggested to do grocery at a particular mall due to weather/traffic conditions, limited available time and past history of user's travel time to that place.

\subsection{Sensors}

Sensors are a popular way to detect various changes in the environment as well as to keep track of user's day to day activities. Our focus is on those sensors that can be worn on the body to identify user's behavior in the form of activities such as, meeting with someone, sleeping, eating, site seeing, etc. Many of these activities are repetitive and if documented properly, they may help depicting the life pattern of the user. Every person is diverse in this World, therefore, it is required to develop such a lifelog system which first learns an individual's living pattern and then based on the past records, makes context based recommendations. In order to record the activities and their context, the sensors that may be used are Bluetooth, NFC, Accelerometer, GPS and Infrared sensors.

Bluetooth sensor which is used for short distance communication, can provide indoor navigation while detecting nearby peoples’ presence [29]. The past records

Mehran University Research Journal of Engineering \& Technology, Volume 36, No. 3, July, 2017 [p-ISSN: 0254-7821, e-ISSN: 2413-7219] 
of a user visiting a Mall or exhibit may help recall the navigation paths and also highlight the booths that were not visited earlier.

NFC allows the mobile devices for TouchMe interaction paradigm, that is, the users can easily establish connections between objects by simply touching them [30]. NFC can be best utilized for authentication, detection of the objects interacted by users and checking in at various points in a city [31]. It can also be used as a medium to collect data from health monitoring devices such as heart rate, glucose and blood pressure monitor [32]. The past records from NFC based sensing may help find misplaced objects like keys, phone, etc. Moreover, the data collected from health monitoring devices may warn the user of any aberrations in bodily functions like destabilized blood glucose, arrhythmia.

The accelerometer sensor, when attached to a device, is capable of observing changes in the $\mathrm{X}, \mathrm{Y}$ and $\mathrm{Z}$ of the device. If this sensor is worn on the body, it can provide useful information such as sleep patterns [33], walking, jogging and cycling [34]. The proposed system may record physical activities and movements made by the users along with the location traces. In future, the lifelog system may be asked for suggestions on walking, jogging and cycling based on the past data and the time taken to perform those activities.
The GPS sensor provides location traces in the form of latitude and longitude coordinates. The user's visited locations can be determined easily with the help of this sensor. The readings from the GPS sensors become more significant if they are fused with the activities performed by the user for instance, user's jogging, walking and cycling track. The frequently visited places where the user stays for longer periods like home, office, and favorite restaurants may infer how the user is spending the day. Thus, the past location traces of the user may be employed to infer the future movement of the user.

The use of body worn sensors along with the IoT aware devices is essential in the proposed lifelog recommendation framework. The lifelog cloud server configured for deploying user's past lifelogs is a necessary step, since it may help to provide the recommendations anywhere and any at any time. We will discuss about the lifelog cloud server (Fig. 1) in the following subsection.

\subsection{Lifelog Cloud}

The lifelog cloud stores the activities performed by the user in the form of activity profiles. It also contains two more components which are activity organizer and activity recommender. The roles of various components of lifelog cloud are discussed in the following subsections.

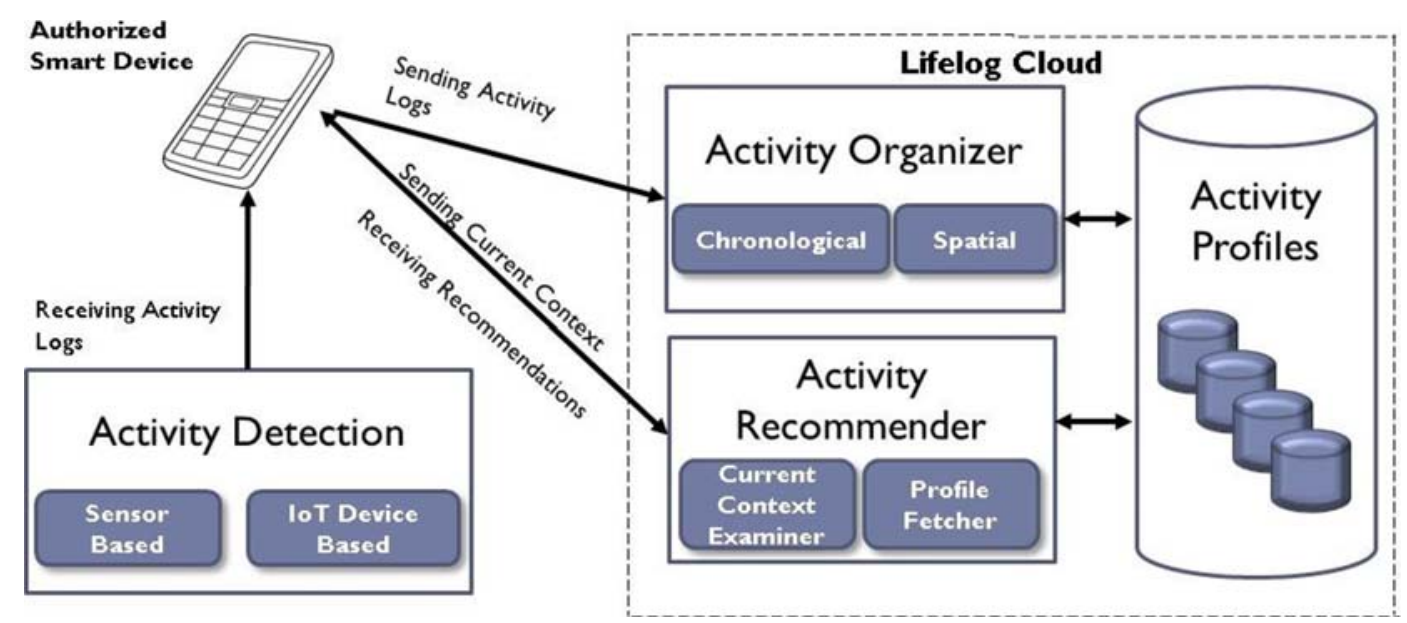

FIG. 1. LIFEREC RECOMMENDATION FRAMEWORK 


\subsubsection{Activity Organizer}

Activity organizer is a part of lifelog cloud where the detected activities or captured lifelogs are managed in chronological as well as spatial order. The Activity Organizer proposed for this system is responsible to receive the lifelogs from the authorized user devices in order to ensure security and privacy. The two significant attributes while recording user activities are location and time. Since, human beings have correlations with time and locations, therefore, the ordering of the activities with respect to time and locations will do a great deal while retrieving the past. For example, the jogging activity time and location is sequenced with the next activity performed by the user after jogging like showering at home. In this manner, on some regular day, if the user wants to go some party after jogging, the user may be recommended accordingly based on the past patterns.

\subsubsection{Activity Profiles}

The activity profiles of an individual user are categorized based on the activities detected by the IoT devices and the sensors worn by the user. For instance, the eating activity profile contains the user's eating patterns collected by the smart fridge as well as the body worn sensors (NFC tags, for instance) for the past several days. Similarly, the watching TV activity profile is loaded with the movies/serials watched by the user in chronological order. These profiles are used by the activity recommender to assist the user for future course of action, like, what type of food may be suitable after consuming the total calories required by a normal person in one day? or what to watch this weekend after viewing an episode of the current season of a serial?. We will further discuss about the activity recommender in the next subsection.

\subsubsection{Activity Recommender}

This is an essential part of the lifelog cloud. Since the lifelogs of the user's past activities are being collected and stored as activity profiles of a user, it is achievable to provide suitable suggestions based on the current situation of the user. The sensors worn by the user and the authorized smart device are capable of providing sufficient context of the user which is passed on to the activity recommender. The module of the activity recommender consists of two parts, current context examiner and profile fetcher. The task of the current context examiner is to determine the past activities that match with the current activity and contextof the user. In the next step, the profiles of similar past activities are fetched from the lifelogcloud. Finally, the activity recommender generates suggestions based on the content based, collaborative and hybrid recommendation approaches [35]. The final recommendations are shown on the user's registered smart device and it is up to the user to follow these recommendations. The cycle of capturing the lifelogs and recommending activities continues to benefit users in their respective lives.

\section{METHODOLOGY}

In this section, we discuss the overall working of the system. The smart device carried by the user not only performs the functionality of authenticating the daily activity lifelogs received from the IoT devices and sensors, but it also fetches recommendations from the lifelog cloud. Fig. 2, shows a sequence diagram, depicting the step by step process of capturing of lifelogs and retrieval of recommendations from the lifelog cloud. The lifelog cloud server organizes the lifelogs and maintains the activity profiles of the user.

We have designed a lifelog application running on an android based smart phone. The application consists of two interfaces, one for authenticating the lifelogs (Fig. 3(a)) and the other for receiving recommendations from the lifelog server (Fig. 3(b)). The user does not necessarily need to authenticate each and every lifelog entity as it is practically impossible. However, the provision of authenticating lifelogs is given for such situations where the user may want to hide those lifelogs which are infringing the privacy. In the current version of the system, we have programmed various sensors for activity detection. The IoT aware device have not been configured in the current version of the system. 
We have used the built in sensors of the smart phone in this prototype which areaccelerometer, Bluetooth, NFC reader, and GPS. In Fig. 4, we show 13 different activities detected by our current prototype system and the sensors involved in detecting those activities.

The activity profiles are created and updated using the MongoDB [36] database which is configured on the lifelog cloud server. The user's smart device automatically sends the lifelogs at the end of the day which are organized and stored at the lifelog cloud server. When the recommendations are requested, the current context of the user is sent to the server. The lifelog cloud server processes the context and sends the recommendations back to the user.

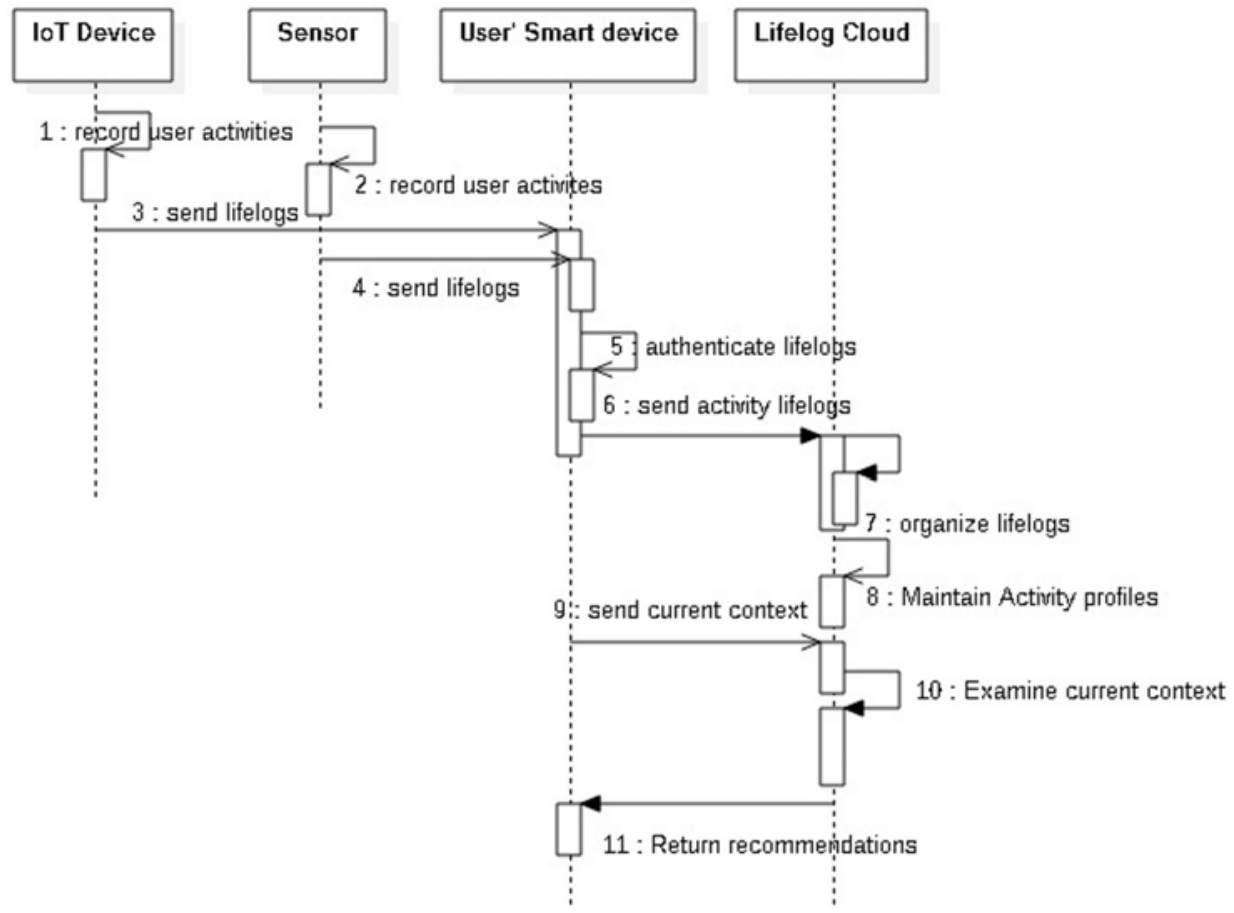

FIG. 2. SEQUENCE DIAGRAM OF THE SYSTEM

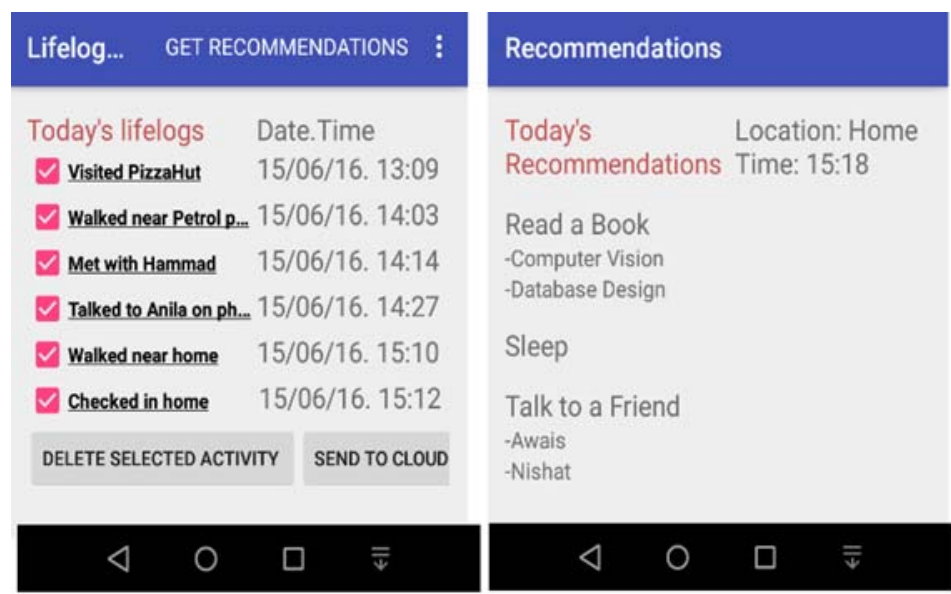

FIG. 3(a). INTERFACE SHOWING ACTIVITY LIFELOGS(LEFT) (b) INTERFACE SHOWING ACTIVITY RECOMMENDATIONS (RIGHT)

Mehran University Research Journal of Engineering \& Technology, Volume 36, No. 3, July, 2017 [p-ISSN: 0254-7821, e-ISSN: 2413-7219] 
The next subsection shows the evaluation results of our prototype system.

\subsection{Evaluation}

We asked three users (aged 24, 27 and 30) to install the system on their smart phones and engage with our application for 5 consecutive days. They had a training session of 2 hours before the start of evaluation. In this session they were briefed about the purpose of this study and working mechanism of the prototype application. The users were able to view lifelog activities and remove any undesirable activity log before being sent to the cloud server. In order to detect 'Reading a book' activity, the users were asked to attach NFC tags to their books. The users may request the application for the recommendations as many times as possible.

The aim of this experiment was to determine the usefulness of our system. We attempted to find whether the recommendations given by the system were followed by the user. The accuracy of activity detection was not observed in this experiment, since many researchers have already proposed algorithms [37,38] for detecting user activities using body worn sensors.

At the end of the experiment, we found that a total of 534 activity profiles were updated at the lifelog cloud server based on the activities of three users for a period of 5 days. The users were given recommendations of 101 activities during the experiment and they followed the suggestions 44 times. In the graph shown in Fig. 5, we compare the average number of activities detected by the proposed system, activities recommended and activities followed by the users. It was observed that 'Talking on phone', 'Meeting with friend' and 'Walking' activities were the most favorite activities of the user as these activities were detected by the system more than other activities. The user acceptance of recommendations was $43 \%$. With these results, we come to the conclusion that the users found the system really useful, since they followed the recommendations and performed their favorite activities repeatedly.

\begin{tabular}{|l|l|}
\hline Walking \\
\hline Sleeping \\
\hline Running \\
\hline Meeting with friend \\
\hline Visiting restaurant \\
\hline Visiting friend's home \\
\hline Visiting agrocery store \\
\hline Checking in a place \\
\hline Working at office \\
\hline Working at home
\end{tabular}

FIG. 4. ACTIVITIES DETECTED BY OUR SYSTEM WITH THE HELP OF SENSORS 


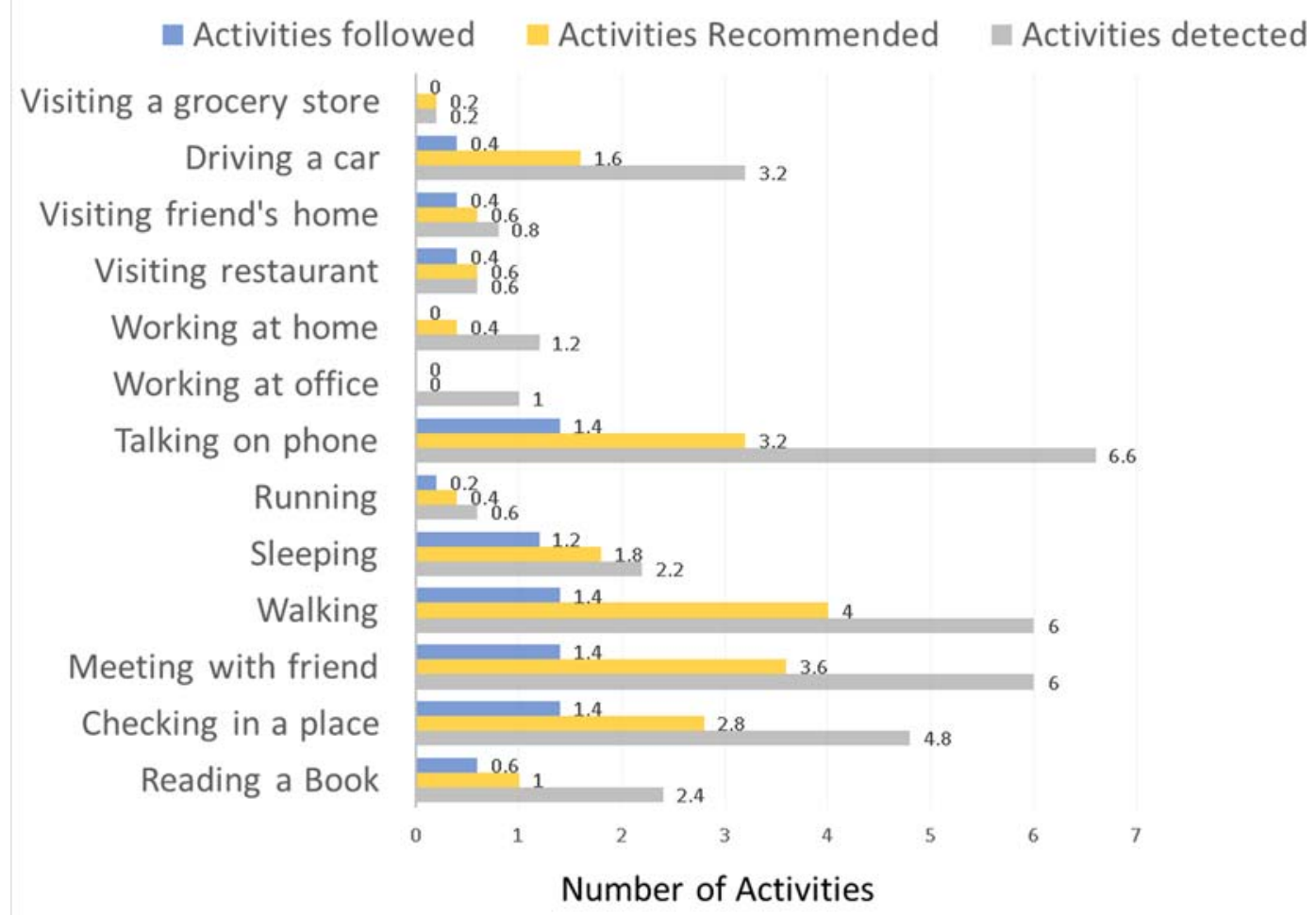

FIG. 5. AVERAGE NUMBER OF ACTIVITIES FOLLOWED, RECOMMENDED AND DETECTED

\section{CONCLUSION}

The proposed LIFEREC framework acquiresactivity information from IoT aware devices and body worn sensors sensors. It maintains activity profiles of various activities performed by the users in their daily lives. The prototype system allows recording of users' activities and generating recommendations based on past recorded data while considering the present situation of the user. These recommendations are displayed on the authorized user device in order to ensure privacy and security. The use of IoT aware devices in this framework is noteworthy since the devices have the ability to record users' interaction and send this data over the network. The LIFEREC framework is extensible and allows addition of sensors that may be used to detect physical activities and current context of the user.

\section{FUTURE WORK}

In future, we will work on the design of applications for IoT devices to obtain activity lifelogs of users and incorporate them for recommendations. We will also work on a friendly user interface that may be employed by the user for viewing the recommendation given by the framework.

\section{ACKNOWLEDGMENTS}

Authors are thankful to Mehran University of Engineering \& Technology, Jamshoro, Pakistan, for provision of facilities for this research. Special thanks to Internal and External Referees/Experts, for their valuable comments and suggestions regarding improvement of the paper.

Mehran University Research Journal of Engineering \& Technology, Volume 36, No. 3, July, 2017 [p-ISSN: 0254-7821, e-ISSN: 2413-7219] 


\section{REFERENCES}

[1] Allen, A.L., "Dredging up the Past: Lifelogging, Memory and Surveillance”, University of Chicago Law Review, Volume 75, No. 1, pp. 47-74, 2008.

[2] Gemmell, J., Bell, G., Lueder, R., Drucker, S., and Wong, C., "MyLifeBits: Fulfilling the Memex Vision", Proceedings of $10^{\text {th }}$ ACM International Conference on Multimedia, pp. 235-238, 2002.

Blum, M., Pentland, A., and Troster, G., "Insense: Interest-Based Life logging”, IEEE Multimedia, Volume 13, pp. 40-48, 2006.

http://www.autographer.com/ (Last Accessed on 30 th August, 2016)

http://getnarrative.com/shop (Last Accessed on 30th August, 2016)

http://www.indiegogo.com/projects/parashoot-smartwearable-video-mini-hd-camera-photo\#/ (Last Accessed on $30^{\text {th }}$ August, 2016)

Kalnikaite, V., Sellen, A., Whittaker, S., and Kirk, D., "Now Let Me See Where I Was: Understanding How Lifelogs Mediate Memory”, Proceedings of 28th International Conference on Human Factors in Computing Systems, pp. 2045-2054, 2010.

Chen, Y., and Jones, J.F., “Augmenting Human Memory using Personal Lifelogs”, Proceedings of $1^{\text {st }}$ ACM International Conference on Augmented Human, Volume 9, 2010.

http://research.microsoft.com/en-us/um/cambridge/ projects/sensecam/ (Last Accessed on 30 $0^{\text {th }}$ August, 2016)

Rawassizadeh, R., and Tjoa, A.M., "Securing Shareable Lifelogs”, Proceedings of IEEE $2^{\text {nd }}$ International Conference on Social Computing, pp. 1105-1110, 2010.

Lee, Y., and Cho, S., "Extracting Meaningful Contexts from Mobile Lifelog”, Proceedings of 8th International Conference on Intelligent Data Engineering and Automated Learning, Springer, pp. 750-759, 2007.
Ohsawa, Y., Benson, N.E., and Yachida, M., “KeyGraph: Automatic Indexing by Co-Occurrence Graph Based on Building Construction Metaphor”, Proceedings of Advances in Digital Libraries Conference, IEEE Computer Society, pp. 12-18, 1998.

[13] Li, Y., and Landay, J.A., “Activity-Based Prototyping of Ubicomp Applications for long-Lived, Everyday Human Activities” Proceeding of $26^{\text {th }}$ Annual SIGCHI Conference on Human Factors in Computing Systems, ACM, pp. 1303-1312, 2008.

[14] Schweer, A., and Hinze, A., "The Digital Parrot: Combining Context-Awareness and Semantics to Augment Memory”, Proceedings of New Zealand Computer Science Research Student Conference, pp. 254-257, 2008.

[15] Satchell, C., Graham, C., and Rouncefield, M., "MoBlogs: Sharing Situations, and Lived Life”, Computer Supported Cooperative Work, Springer, pp. 269-289, 2009.

[16] Memon, M.A., Bhatti, S., and Mahoto, N.A., "A Digital Diary: Remembering the Past Using the Present Context”. Mehran University Research Journal of Engineering \& Technology, Volume 35, No. 2, pp. 275-286, Jamshoro, Pakistan, April, 2016.

[17] Memon, M.A., Mahoto, N.A., Khuhawar, F.Y, and Tanaka, J. "Retrieval of Life Logs based on Users Context”, Sindh University Research Journal (Science Series), Volume 47, No. 4, pp. 851-860, Jamshoro, Pakistan, 2015.

[18] Kumar, G., Jerbi, H., Gurrin, C., and O’Mahony, M.P., “Towards Activity Recommendation from Lifelogs", Proceedings of $16^{\text {th }}$ International Conference on Information Integration and Web-Based Applications \& Services, ACM, pp. 87-96, 2014.

[19] Tezuka, H., Ito, K., Murayama, T., Seko, S., Nishino, M., Muto S., and Abe, M., "Restaurant Recommendation Service Using Lifelogs”, NTT Technical Review, Volume 9, No. 1, 2011.

[20] Lin, Y., Jessurun, J., Vries, B.D., and Timmermans, H., "Motivate: Context Aware Mobile Application for Activity Recommendation”, Proceedings of $2^{\text {nd }}$ International Conference on Ambient Intelligence, Springer, pp. 210-214, 2011. 
[21] He, Q., and Agu, E., “On11: An Activity Recommendation Application to Mitigate Sedentary Lifestyle”, Proceedings of Workshop on physical Analytics, pp. 3-8, 2014.

[22] Atzori, L., Iera, A., and Morabito, G., "The Internet of Things: A Survey”, Elsevier Computer Networks, 2010.

[23] ITU Internet Reports, "The Internet of Things", November, 2005.

[24] Zorzi, M., Gluhak, A., Lange, S., and Bassi, A., "From Today's INTRAnet of Things to a Future INTERnet of Things: A Wireless and Mobility-Related View”, IEEE Wireless Communications, Volume 17, No. 6, pp. 44-51, 2010

Sandholm, T., Lee, D., Tegelund, B., Han, S., Shin, B., and Kim, B., "CloudFridge: A Testbed for Smart Fridge Interactions”, Work Supported by the IT R\&D Program of MSIP/KEIT, 2014.

Sony Global Press Release, "Philips and Sony Announce Strategic Cooperation to Define Next Generation Near Field Radio-Frequency Communications, 5ht September, 2002, Retrieved from http://www.sony.net/SonyInfo/ News /Press_Archive/200209/02-0905E/ (Last Accessed on 30th August,, 2016)

Loukadaki, M., and Edwards, T., “CAN Signal Injection”, IEEE International Conference on Vehicular Electronics and Safety, pp. 68-71, 2012.

Li, S., Li, K., Rajamani R., and Wang, J., "Multi-Objective Coordinated Control for Advanced Adaptive Cruise Control System", Proceedings of 48th IEEE Conference on Decision and Control, pp. 3539-3544, 2009.

[29] Altini, M., Brunelli, D., Farella, E., and Benini, L., "Bluetooth Indoor Localization with Multiple Neural Networks", $5^{\text {th }}$ IEEE International Symposium on Wireless Pervasive Computing, pp. 295-300, 2010.

[30] Pohjanheimo, L., Keränen, H., and Ailisto, H., "Implementing Touchme Paradigm with a Mobile Phone”, Proceedings of Joint Conference on Smart Objects and Ambient Intelligence: Innovative ContextAware Services: Usages and Technologies, ACM, pp. 87-92, 2005.
Toto, S., "Via NFC: Japanese Social Network Mixi First to Let Users "Share” Real-World Items", Technology Crunch, Retrieved from https://techcrunch.com/2011/ 02/11/via-nfc-japanese-social-network-mixi-first-to-letusers-like-real-world-items/ (Last Accessed on 30 ${ }^{\text {th }}$ August, 2016)

Strommer, E., Kaartinen, J., Parkka, J., Ylisaukko-Oja A., and Korhonen, I., "Application of Near Field Communication for Health Monitoring in Daily Life”, 28th Annual IEEE International Conference on Engineering in Medicine and Biology Society, pp. 3246-3249, 2006.

Chen, Z., Lin, M., Chen, F., Lane, N.D., Cardone, G., Wang, R., Li, T., Chen Y., Choudhury, T., and Campbell, A.T., "Unobtrusive Sleep Monitoring Using Smartphones", 27th International Conference on Pervasive Computing Technologies for Healthcare and Workshops, pp. 145-152, 2013.

Bonomi, A.G., Goris, A.H., Yin, B., and Westerterp, K.R., "Detection of Type, Duration, and Intensity of Physical Activity using an Accelerometer”, Medical Science Sports Exercise, Volume 41 No. 9, pp. 1770-1777, 2009.

[35] Li, Q., and Kim, B.M., “An Approach for Combining Content-Based and Collaborative Filters”, Proceedings of $6^{\text {th }}$ International Workshop on Information Retrieval with Asian Languages, pp. 17-24, 2003.

[36] https://www.mongodb.com/ (Last Accessed on 22 $2^{\text {nd }}$ October, 2016)

[37] Torres-Huitzil, A., and Alvarez-Landero “Accelerometer Based Human Activity Recognition in Smartphones for Healthcare Services", Mobile Health Springer Series in Bio-Neuroimformatics Volume 5, pp. 147-169 2015.

[38] Liu, L., Peng, Y., Liu, M., and Huang, Z., "Sensor-Based Human Activity Recognition System with a Multilayered Model using Time Series Shapelets”, Knowledge-Based Systems, Volume 90, pp. 138-152, 2015. 